\title{
Charge transport through carbon nanotubes interacting with light
}

\author{
Kannan Balasubramanian and Marko Burghard \\ Max-Planck-Institut für Festkörperforschung, Heisenbergstr. 1, D70569 Stuttgart, Germany \\ E-mail: b.kannan@fkf.mpg.de and m.burghard@fkf.mpg.de
}

Received 1 March 2006, in final form 23 April 2006

Published 17 October 2006

Online at stacks.iop.org/SST/21/S22

\begin{abstract}
This paper focuses on photoconductivity and electroluminescence studies on individual single-wall carbon nanotubes (SWCNTs) that are incorporated into nanoscale electrical devices. It describes how the spatial dependence of these signals has been utilized to gain valuable information about the local electronic structure and the charge transport properties of the nanotubes. Moreover, we present a summary of available experimental data supporting theoretical predictions, that excitonic effects dominate the optical absorption and emission of semiconducting SWCNTs. Finally, an outline of the emerging field of SWCNT-based photovoltaic devices is given, followed by an outlook into the future perspective of carbon nanotube optoelectronics.
\end{abstract}

(Some figures in this article are in colour only in the electronic version)

\section{Introduction}

Single wall carbon nanotubes (SWCNTs) are an intriguing class of materials, whose applications range from mechanically reinforced composites [1] to biosensors [2]. They are evolving to be promising components of nanoscale electronic devices [3]. Towards fabrication of such devices, a detailed understanding of the tubes' electronic structure and charge transport properties is essential. The observation of phenomena such as Coulomb blockade, Luttinger liquid behaviour and Kondo effect in SWCNTs has made them ideally suited for studying mesoscopic physics in one dimension [4]. From an application perspective, a number of factors govern the performance of SWCNT-based devices, including the type of contacts, the presence of structural inhomogeneities as well as the nature of the raw material used. A detailed understanding of the impact of these factors on the electronic properties of SWCNTs is, however, still in its infancy. While much has been learnt about the electrical transport properties of SWCNTs in the dark, charge transport studies through SWCNTs interacting with light have only recently been pursued. Nonetheless, the latter investigations have important implications for the design of future nano-scale optoelectronic, photovoltaic and electro-optical nanotube-based devices.

This paper is organized as follows. After a short introduction of the electronic structure of SWCNTs, photodesorption effects upon UV illumination of SWCNTs are discussed. Next, a chronological collection of photoconductivity experiments performed on SWCNTs is given. Spatially resolved photoelectronic transport studies are presented in the next section. The following section is devoted to various optical spectroscopy studies demonstrating that absorption in semiconducting SWCNTs is dominated by excitonic effects. Apart from electronic transport measurements under photo-illumination, further experiments have addressed electroluminescence phenomena in SWCNT devices, as outlined in the next section. Following this, photovoltaic devices comprising pristine SWCNTs as well as SWCNT-based hybrid systems are presented. The paper concludes with currently unresolved issues and future perspectives.

\section{Fundamentals}

Carbon nanotubes — denoted by chiral indices $(n, m)$ — can be classified as armchair $(n=m)$, zigzag $(m=0)$ and chiral $(n \neq$ $m, m \neq 0)$. In a first approximation, their electronic structure can be derived from that of graphene by the zone-folding approach [5]. In general, $(n, m)$ nanotubes with $n-m$ being a multiple of 3 are metallic, while the rest are semiconducting. Figure 1 displays the electronic density of states (EDOS) of the $(15,0)$ metallic and $(14,0)$ semiconducting SWCNTs, both of which exhibit pronounced peaks or van Hove singularities 

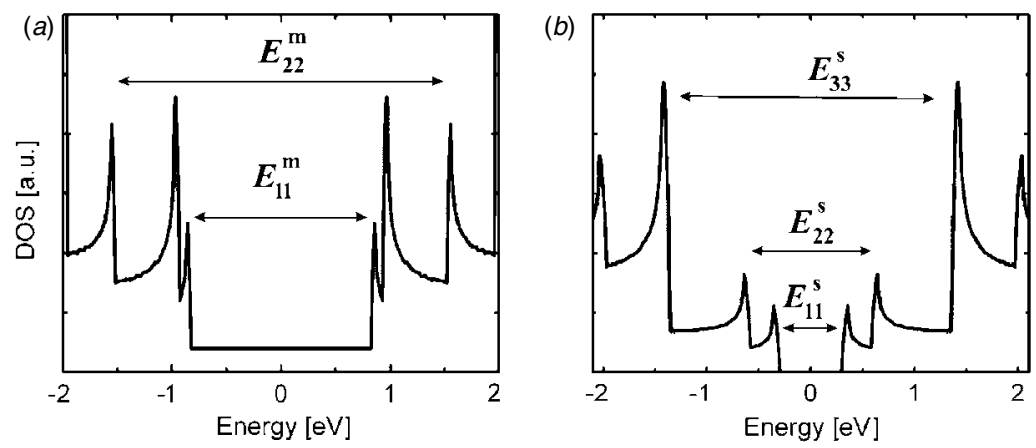

Figure 1. Calculated electronic density of states (EDOS) of carbon nanotubes: $(a)$ EDOS of a $(15,0)$ metallic SWCNT and $(b)$ EDOS of a $(14,0)$ semiconducting SWCNT. A value of $-2.9 \mathrm{eV}$ was used for the tight binding transfer integral. $E_{11}{ }^{\mathrm{m}}$ for the $(15,0)$ tube exhibits a splitting due to the trigonal warping effect.

(vHs) characteristic of their quasi-1D nature. The series of interband transitions, each of them comprising the energy difference between a pair of vHs, is represented by $E_{i i}{ }^{x}$, where $i$ denotes the number of the EDOS peak pair and $x$ is either ' $\mathrm{m}$ ' or 's' for metallic (m-) or semiconducting (s-) SWCNTs, respectively. The band gap of s-SWCNTs is calculated as $E_{11}{ }^{\mathrm{s}} \approx 0.74 \mathrm{eV} / d$, while $E_{11}{ }^{\mathrm{m}}$ for $\mathrm{m}-\mathrm{SWCNTs}$ is $\approx 2.22 \mathrm{eV} / d$, where $d$ is the diameter of the nanotube in nanometres [6]. The SWCNTs used in experimental studies vary in diameter depending on their method of production.

Most of the experimental charge transport studies to be discussed here involve the use of a field-effect transistor (FET) configuration incorporating either a single SWCNT or networks of SWCNTs as the channel. The substrate is usually highly doped silicon with a thermally grown $\mathrm{SiO}_{2}$ layer (100 to $500 \mathrm{~nm}$ thickness) as gate insulator. The spacing between the drain and the source electrodes can vary from $100 \mathrm{~nm}$ up to $100 \mu \mathrm{m}$. Transport characteristics are studied by applying a combination of drain-source bias $\left(V_{\mathrm{ds}}\right)$ and gate voltage $\left(V_{\mathrm{G}}\right)$ and measuring the current $\left(I_{\mathrm{d}}\right)$ flowing through the channel. While for $\mathrm{m}-\mathrm{SWCNTs} I_{\mathrm{d}}$ remains constant as a function of $V_{\mathrm{G}}$, s-SWCNTs show a strong modulation in their conductance of up to six orders of magnitude. The fabricated s-SWCNT-FETs can be classified into two different categories. As-produced s-SWCNT-FETs that are not subjected to any further treatment display p-type behaviour, i.e., they show high drain currents (ON state) at negative $V_{\mathrm{G}}$ and low currents (OFF state) at positive $V_{\mathrm{G}}$. This property has been attributed to hole-doping of the nanotubes and/or the nanotube-metal contacts by oxygen physisorption under ambient conditions $[7,8]$. The Schottky barriers at the contacts in these devices are such that hole injection is favoured [9]. A second kind of s-SWCNT-FET devices show ambipolar characteristics with high currents at both positive and negative $V_{\mathrm{G}}$, and low currents at intermediate $V_{\mathrm{G}}$. These devices can for instance be prepared using $\mathrm{Ti}$ electrodes that are annealed in an inert atmosphere followed by covering with a protective layer [10]. It has been suggested that annealing leads to the formation of $\mathrm{TiC}$ at the tube/contact interface that provides for a thin and low Schottky barrier, whereby the injection of both holes and electrons into the nanotube channel becomes possible.

\section{Photodesorption}

The electronic transport properties of an individual s-SWCNT (p-type) under photo-illumination were first reported by Chen et al [11], who used a chemical vapour deposition (CVD)grown tube with a diameter of $1.5 \mathrm{~nm}$ and contacted by electrodes spaced $\approx 1.5 \mu \mathrm{m}$ apart. Upon global illumination of the sample by UV light $\left(\lambda_{\text {exc }}=254 \mathrm{~nm}\right)$ with a relatively low laser intensity of $2 \mathrm{~mW} \mathrm{~cm}{ }^{-2}$, a decrease in conductance was observed. This change was ascribed to photo-induced desorption of oxygen from the surface of the s-SWCNT, which causes a reduction of hole carriers and hence a lowering of the sample conductance. As the high energy of illumination $(4.9 \mathrm{eV})$ is close to the work function of the s-SWCNT ( $\approx 5 \mathrm{eV}$ for the investigated s-SWCNT) [12], the photo-excited electrons have a high probability of inducing such a molecular desorption process. The wavelength dependence of the photo-induced current change revealed that photodesorption is maximal at high energies and reduces dramatically towards lower excitation energies. On this basis, the authors attributed the photo-induced desorption to the excitation of $\pi$-plasmons in the tube. A similar conclusion was drawn for a SWCNT network sample.

Support for photo-induced desorption of oxygen has also been gained by a subsequent study [13]. Here, the transport characteristics of individual p-type s-SWCNTs obtained from various growth procedures were investigated in a systematic manner. The tubes were contacted by different metals ( $\mathrm{Ti}$, $\mathrm{Au}$ or Pd) to identify if the photo-current decrease was due to the contacts or the tubes themselves. Upon excitation with UV light $\left(\lambda_{\text {exc }} \approx 340 \mathrm{~nm}\right.$ ), the authors observed a large photocurrent decrease in samples contacted with $\mathrm{Ti}$, in comparison to no or little change in the case of $\mathrm{Au}$ or $\mathrm{Pd}$. The reduction in photo-current in the former case could be correlated with an increase in the Schottky barrier heights, caused by the photodesorption of oxygen from the surface oxide layer at the contacts. The little or no photo-induced current change for the latter case was attributed to the comparably low photodesorption cross sections for $\mathrm{Au}$ and $\mathrm{Pd}$ with respect to $\mathrm{TiO}_{2}$.

\section{Photoconductivity}

Photoconductivity in s-SWCNTs has been observed in a number of experiments utilizing laser excitation of lower 
energy than UV radiation. Initial studies in this direction concentrated on networks of SWCNTs containing both m- and s-SWCNTs. For instance, Fujiwara et al [14] used a pulsed laser with energies tuneable in the range of 0.5 to $2.8 \mathrm{eV}$ to illuminate $300-500 \mathrm{~nm}$ thick SWCNT films contacted by gold electrodes separated $10 \mu \mathrm{m}$ apart. The photo-current at room temperature showed two strong peaks at excitation energies of 0.7 and $1.2 \mathrm{eV}$, in agreement with the first two interband transitions $E_{11} \mathrm{~s}$ and $E_{22}$ s for the arc discharge tubes used in that study. In subsequent experiments with SWCNT films of similar thickness, photoconductivity could be observed at nearinfrared wavelengths corresponding to an energy of $\approx 1.46 \mathrm{eV}$ [15]. The samples exhibited a slow response of the photocurrent, which was attributed to the presence of adsorbed oxygen on the SWCNT films.

While the origin of photoconductivity observed in the above experiments could not be clarified further due to the use of a mixture containing both m-SWCNTs and s-SWCNTs, this task could be achieved through the investigation of single nanotubes. Freitag et al [16] examined the photo-response of an individual ambipolar s-SWCNT-FET by global illumination of the device in the OFF state with a laser tuneable in the energy range of 1.27 to $1.59 \mathrm{eV}$. The photo-current showed a linear dependence on the laser intensity and was maximum for the light polarization being parallel to the orientation of the tube. At a laser intensity of $1 \mathrm{~kW} \mathrm{~cm}{ }^{-2}$, a photoconductance of $\approx 5 \mathrm{pS}$ was observed. The authors proposed a mechanism wherein the incident infrared light resonantly excites the $E_{22}{ }^{\text {s }}$ transition of the s-SWCNT. The energy dependence of the photo-current (photo-current spectra) of various samples displayed a resonance peak in the range of 1.27 to $1.5 \mathrm{eV}$ in agreement with the expected $E_{22}$ s for the SWCNTs used there.

Photoconductivity from an individual nanotube implemented into a p-type s-SWCNT-FET has been observed by exciting the $E_{33}{ }^{\mathrm{s}}$ transition [17]. The source of light was a diffraction-limited laser spot (diameter $\approx \lambda_{\text {exc }} / 2$ ) obtained from a confocal laser scanning microscope. Upon illumination of the tube in the OFF state with $\lambda_{\text {exc }}=514.5 \mathrm{~nm}$ $(2.4 \mathrm{eV})$, a pronounced increase in current was observed as shown in figure 2(a). The photo-response was linear within a laser intensity range of 0.5 to $7.5 \mathrm{MW} \mathrm{cm}^{-2}$. A photoconductance of at least $2 \mathrm{nS}$ was measured at an incident laser intensity of $1 \mathrm{MW} \mathrm{cm}$. It is noteworthy that the intensity used was three orders of magnitude higher than that for the ambipolar device of Freitag et al [16]. Moreover, the photo-response remained unaffected when the environment was changed from air to oxygen, argon or water vapour. The assertion that photo-generated charge carriers in the nanotube are responsible for the current increase, gained further support by the observation of maximal photoconductance for light polarization along the tube axis, as illustrated in figure $2(b)$.

It is worthwhile to take a look at the effect of photoillumination on the gate-dependence of conductance as obtained from the above two experiments. Figure 3(a) shows the gate characteristics with and without global infrared illumination for the ambipolar device [16]. The gate dependence for the p-type device with and without local visible light irradiation is displayed in figure $3(b)$. In both cases, a sizeable photo-current that is distinguishable from the dark current can mainly be observed in the low
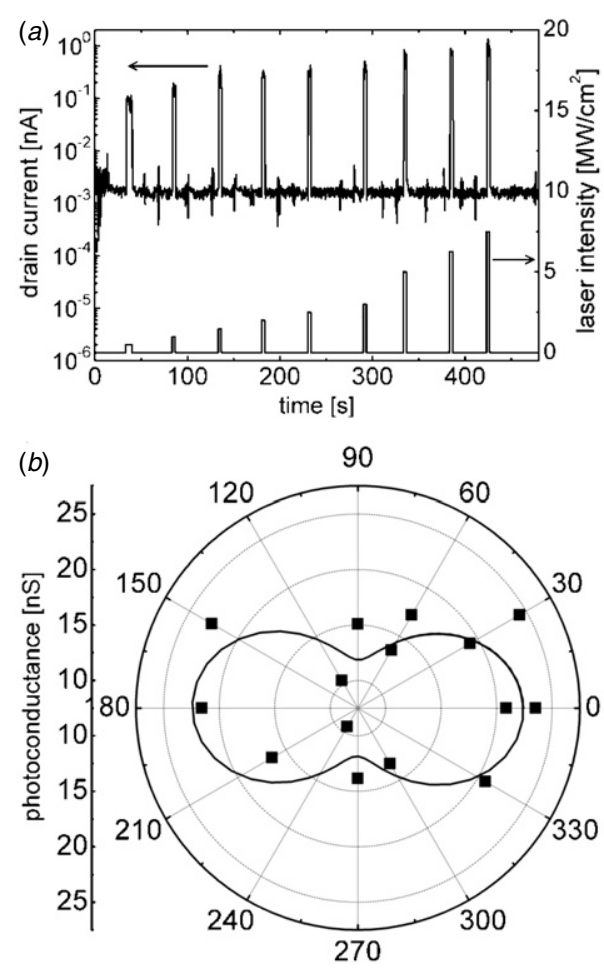

Figure 2. (a) Room temperature currents (plotted in log scale) measured on a single semiconducting $\operatorname{SWCNT}\left(V_{\mathrm{ds}}=0.1 \mathrm{~V}\right)$ upon illumination at a laser wavelength of $\lambda_{\text {exc }}=514.5 \mathrm{~nm}$ at various laser intensities with pulse widths of 3 or $7 \mathrm{~s}$. (b) Polarization dependence of the photoconductance of the SWCNT, where $0^{\circ}$ corresponds to parallel orientation between the tube and the light polarization. Adapted from [17].

current or OFF state gate voltage regimes. By contrast, the photodesorption measurements outlined in the previous section [11] were performed within the ON state of the s-SWCNT. Under such a condition, the photo-induced carriers contribute only little to the charge transport, possibly explaining why photoconductivity could not be measured in this experiment.

\section{Photoelectronic transport imaging}

Charge transport through SWCNT-based devices is often dominated by potential barriers at the contacts and at defect sites along the tubes. Thus, information about the local electronic structure of SWCNTs is vital for identifying the factors that limit the performance of nanotube-based electrical devices. One possibility to obtain such information is to study the effect of local illumination on the charge transport through individual SWCNTs. With the aid of a diffraction-limited laser spot, it has been possible to measure the photo-current signal originating from illumination at different locations along individual tubes, with a resolution of $\approx \lambda_{\text {exc }} / 2$. In such an experiment, the sample is scanned below the stationary laser spot and the photo-induced current signal at a certain drainsource bias is recorded as a function of the sample position, to obtain a photoelectronic transport (PET) image [17, 18]. 

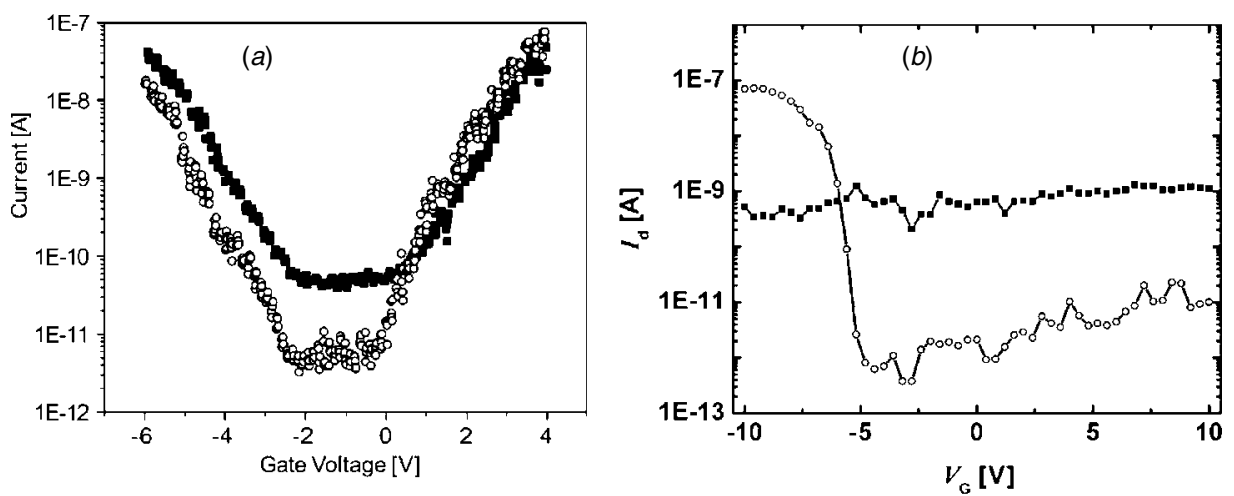

Figure 3. Gate dependence of drain current for a s-SWCNT-FET device with and without laser illumination: $(a)$ ambipolar device with infrared illumination $\left.(1.2 \mathrm{eV}, 1 \mathrm{~kW} \mathrm{~cm})^{-2}\right)$ at $V_{\mathrm{ds}}=0.5 \mathrm{~V}$. Reprinted with permission from [16]. () 2003 American Chemical Society. (b) p-type device with $\lambda_{\mathrm{exc}}=514.5 \mathrm{~nm}\left(2.4 \mathrm{eV}, 0.5 \mathrm{MW} \mathrm{cm}^{-2}\right)$ at $V_{\mathrm{ds}}=0.1 \mathrm{~V}$. Open circles and solid squares correspond respectively to data measured in the dark and under illumination.

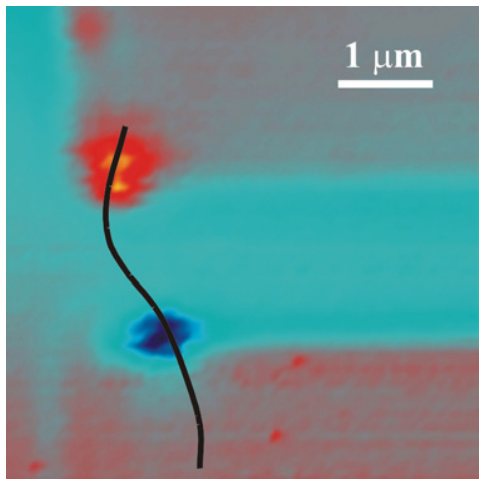

Figure 4. Photoelectronic transport image of a p-type s-SWCNT device plotting the drain current at zero drain-source bias with $V_{\mathrm{G}}=$ $0\left(\lambda_{\text {exc }}=514.5 \mathrm{~nm}, 0.5 \mathrm{MW} \mathrm{cm}^{-2}\right)$. A simultaneously taken reflection image is superposed to obtain the approximate position of the electrodes and nanotube. The contacted nanotube is drawn with a thick black line as obtained from an AFM image. The current is positive at the bright red spot and negative at the dark blue spot, while the background current is zero.

\subsection{Schottky barriers in s-SWCNTs}

Figure 4 shows a PET image acquired from a p-type sSWCNT-FET at zero drain-source bias upon illumination at $\lambda_{\text {exc }}=514.5 \mathrm{~nm}$. The image displays two strong oppositely signed lobes close to the two contacts, which indicate high short-circuit photo-currents. At the contacts, the nanotube electronic bands are bent leading to the creation of an internal electric field. The short-circuit photo-current is attributed to the generation of an offset photo-voltage at these Schottky contacts, similar to the case of conventional metal-semiconductor Schottky junctions [19]. The opposite polarities of the photo-current signals are due to the mutually opposite orientations of the band bending profiles at the two contacts. In comparison, due to the flat bands in the middle of the tube, no photo-current is generated in this region.

A simple model for the electronic transport through sSWCNTs under local illumination is schematically sketched in figure 5. Figure 5(a) displays the case of illuminating the tube region in between the contacts. In the absence of an applied bias, the photo-generated electrons and holes do not
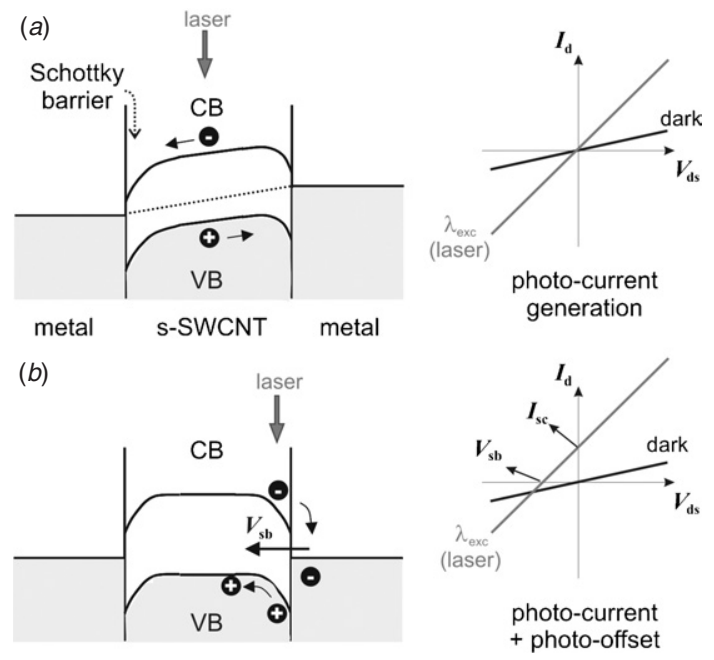

Figure 5. A model of photoelectronic transport through s-SWCNTs. (a) Illumination in the middle of the gap. Left: upon illumination the photo-generated carriers drift with the applied bias. Right: schematic $I-V$ curves showing the dark current and the photo-current. (b) Illumination at the contacts. Left: an offset photo-voltage is generated at the Schottky contacts due to the local band bending. Right: corresponding $I-V$ curves in the dark and upon photo-illumination showing $I-V$ curve offset by $V_{\mathrm{sb}}$. The short-circuit photo-current is denoted as $I_{\text {sc. }}$. Note that the band structure in $(a)$ is shown for $V_{\mathrm{ds}} \neq 0$, while that in $(b)$ is depicted for $V_{\mathrm{ds}}=0$. CB stands for conduction band and VB for valence band.

contribute to a current due to the flat bands here. When a bias is applied, the charge carriers drift due to the electric field and generate a current. The $I-V$ curve upon illumination thus shows an increase in slope corresponding to photoconductivity through the s-SWCNT, as observed in the experiments $[16,17]$. Figure $5(b)$ depicts the situation when the contact region is illuminated. In this case, even without application of a bias voltage, the photo-generated carriers are separated due to the local band bending at the Schottky contacts. This leads to the creation of an offset photo-voltage $\left(V_{\mathrm{sb}}\right)$. The $I-V$ curve in this situation has the same slope as in figure 5(a) but is offset by $V_{\mathrm{sb}}$. Thus, illumination of the contacts results in the generation of a current (equal to $I_{\mathrm{sc}}$ ), accounting for the two spots in figure 4. 

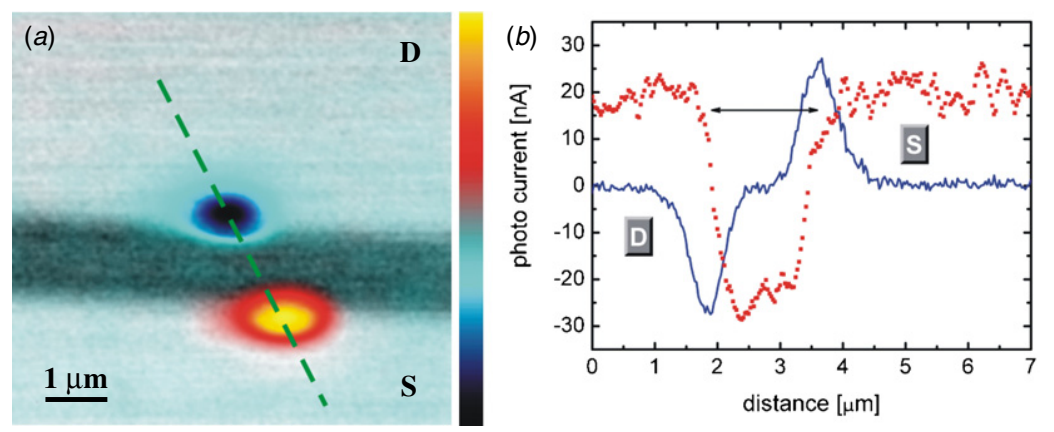

Figure 6. (a) Photoelectronic transport (PET) image of an individual $\mathrm{m}$-SWCNT at zero bias. The image is superimposed on a simultaneously recorded reflection image. The dashed line indicates the position of the nanotube as obtained from an AFM image. ( $\mathrm{S}$ - source, D-drain, $\lambda_{\mathrm{exc}}=514.5 \mathrm{~nm}, 0.5 \mathrm{MW} \mathrm{cm}{ }^{-2}$ ). The colour bar shows a range of -27 to $+27 \mathrm{nA}$. $(b)$ The blue solid line and the red dotted line show respectively the line profiles of the photo-current and the reflection signal along the green dashed line marked in the PET image. The double-sided arrow marks the gap region between the electrodes. Reprinted in part with permission from [18]. @ 2005 American Chemical Society.

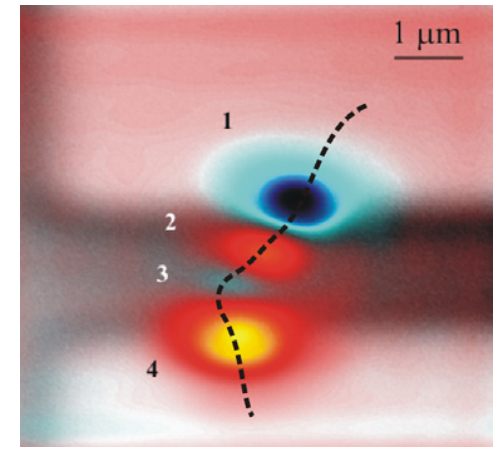

Figure 7. PET image of a defective $\mathrm{m}-\mathrm{SWCNT}$ at $\lambda_{\mathrm{exc}}=514.5 \mathrm{~nm}$ $\left(0.5 \mathrm{MW} \mathrm{cm}^{-2}\right)$. The position of the SWCNT extracted from an AFM image is shown with a dashed line. The image also includes an overlay of a reflection image where the extension of electrodes can be clearly identified. Apart from the two main lobes at the contacts, two additional weaker lobes along the tube can be identified. The colour scale is similar to that used in figure 6 with a range of -3 to $+3 \mathrm{nA}$. Reprinted with permission from [18]. (c) 2005 American Chemical Society.

\subsection{Charge transport barriers in $m-S W C N T s$}

Similar to s-SWCNTs, the electronic transport through mSWCNTs is also affected by the contacts and defects, making the investigation of their spatially resolved photoelectronic transport equally important. In such experiments, the devices have been classified into two categories [18]: type I devices comprising individual defect-free m-SWCNTs, and type II devices containing m-SWCNTs with structural inhomogeneities. The defect-free m-SWCNT devices exhibited a room-temperature conductance close to $e^{2} / h$ and almost no gate dependence of conductance. On the other hand, the conductances of the type II devices were much lower than $e^{2} / h$ and exhibited a p-type gate dependence, with the ratio of the ON to OFF current ranging from 3 to 10, suggestive of defects present along the tube [20].

Figure 6(a) shows a typical PET image obtained from an individual type I m-SWCNT at zero bias, using an excitation wavelength of $514.5 \mathrm{~nm}$, close to $E_{11}{ }^{\mathrm{m}}$ of the investigated tube. Two oppositely signed lobes are clearly visible at the contacts. This can also be inferred from the line profiles of the PET image shown in figure $6(b)$. These lobes exhibit similar

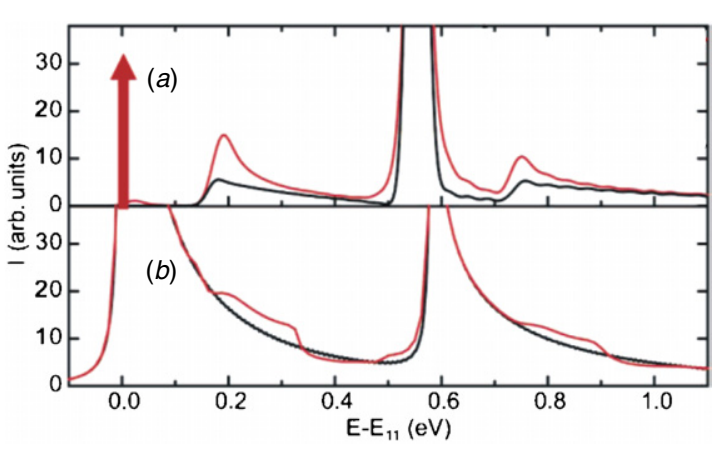

Figure 8. (a) Absorption spectra of a $(17,0)$ nanotube calculated with (red line) and without (black) exciton-phonon coupling in a dielectric environment of $\varepsilon=4$. The vertical arrow at zero energy marks the onset of the first optically active exciton. (b) Absorption spectra of the same tube with (red) and without (black) electron-phonon coupling in an inter-band absorption model. Reprinted with permission from [39]. (C) 2005 American Chemical Society.

behaviour to those observed in PET images of s-SWCNTs (cf figure 4). Specifically, the signs of the two lobes interchange when source and drain electrodes are exchanged. Moreover, $I-V$ curves taken with the laser spot at the positions of the lobes reveal that the short-circuit current originates from an offset photo-voltage. Accounting for the resistance of the m-SWCNT, the associated voltage offset is calculated to be $\approx 0.2 \mathrm{mV}$, about three orders of magnitudes lower than that for s-SWCNTs. The photo-currents generated at the metal contacts reflect the presence of Schottky-like barriers in the m-SWCNTs. Their formation can be understood in terms of the charge transfer occurring when the tube comes into contact with the metal electrode, keeping in mind that the $1 \mathrm{D} / 3 \mathrm{D}$-characteristic of this contact region [21] differs from a simple 3D/3D metal interface. An additional contribution may arise from symmetry breaking of the m-SWCNT, due to the top metal atoms in the contact region. According to theory, the breaking of nanotube symmetry results in the formation of a potential barrier of around $0.1 \mathrm{eV}[22,23]$, rendering the tube locally semiconducting [24]. The identical magnitude of the photo-currents generated at the two contacts of the tube in figure 6 implies the presence of symmetric contact barriers. Some samples, however, displayed considerably 
different magnitudes of the peak photo-current, indicating the presence of asymmetric contacts, similar to observations made on s-SWCNTs [25].

PET images obtained from type II samples show, in addition to the two lobes at the contacts, extra lobes between the contacts. These lobes display a variety of patterns with respect to the sign of the photo-current. Figure 7 shows an example of a PET image obtained from a type II mSWCNT at zero bias with $\lambda_{\mathrm{exc}}=647.1 \mathrm{~nm}$. In addition to the contact lobes, two more lobes marked as ' 2 ' and ' 3 ' are recognizable, reflecting the presence of local builtin electric fields along the tube. For the specific case of the tube in figure 7, the two additional lobes have opposing signs. One possible explanation for this feature is that the tube is composed of a central semiconducting segment connecting two metallic segments via structural defects [26, 27]. The two metal-semiconductor junctions would then behave like Schottky-barriers responsible for the creation of lobes 2 and 3. Alternatively, a strong scattering site could be present at each of the spots 2 and 3, locally introducing potential barriers [28, 29] or metal-semiconductor transitions [30] and associated built-in electric fields [31]. Further systematic studies, supported by complementary experiments such as conducting tip AFM, should lead to an improved understanding of the influence of defects on the local electronic structure of m-SWCNTs.

\section{Nature of excited states}

Initially, the interband transitions underlying photoconductivity in s-SWCNTs have been interpreted within the framework of the simple one-electron picture [5]. However, a series of more recent experiments have revealed the prominence of strong Coulomb interaction between the photo-excited electron and hole, leading to highly correlated electron-hole paired states in the form of excitons. Using two-photon excitation spectroscopy [32] and ultrafast pumpprobe spectroscopy [33], exciton binding energies as high as $400 \mathrm{meV}$ have been reported for nanotubes. The typical separation between the electron and hole in the correlated exciton state is estimated to be a few nm [34]. Compared to the quasi-1D nanotubes, excitonic effects can be largely neglected in bulk semiconductors at room temperature, due to their much lower exciton binding energies of the order of a few meV. The greater importance of excitonic effects in nanotubes is due to enhanced Coulomb interactions. This is a consequence of shorter distances between electrons and holes, that exist in the reduced phase space of the $1 \mathrm{D}$ nanotube system. Added to this, the dielectric screening is greatly reduced, since the electric field lines from the electron-hole pair lie largely outside the nanotube.

Support for the existence of excitons in nanotubes has also been gained by electroabsorption (EA) spectroscopy $[35,36]$. This technique, which involves recording the optical absorption of the material as a function of the applied electric field $F_{\text {app }}$, is well established for organic and inorganic semiconductors. For example, in the case of polydiacetylene, it has been possible to discriminate between the absorption due to quasi-1D excitons from the one due to the continuum [37]. Excitons are known to exhibit a quadratic Stark effect,

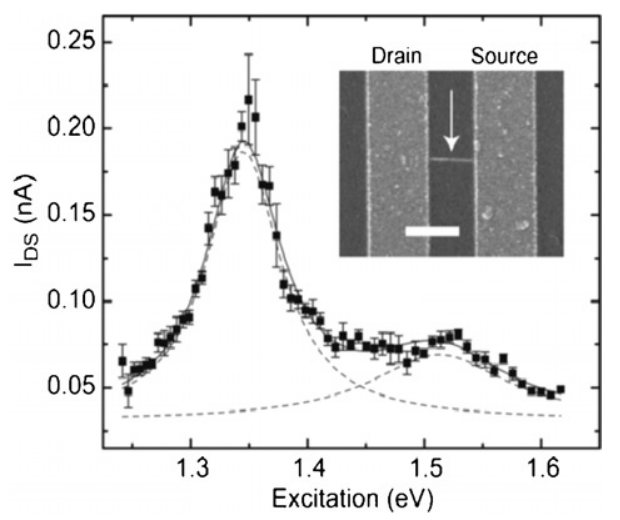

Figure 9. Photo-current spectra of an individual CNT-FET acquired with the light polarization parallel to the orientation of the CNT. The drain-source bias is $-1 \mathrm{~V}$, while the gate voltage is $-0.5 \mathrm{~V}$. The dotted lines are Lorentzian fits to the measured data. The inset shows an SEM image of the investigated CNT-FET. The scale bar is $1 \mu \mathrm{m}$. Reprinted with permission from [39]. (c) 2005 American Chemical Society.

i.e. the EA signal scales as the square of $F_{\text {app. }}$ By contrast, the EA signal due to free electrons shows Frank-Keldysh (FK)-type oscillations in energy corresponding to a $\left(F_{\text {app }}\right)^{1 / 3}$ response. EA spectra have been measured on HiPco SWCNTs embedded in a transparent polymer matrix under an external electric field in the range of 0.5 to $2 \mathrm{MV} \mathrm{cm}^{-1}$. The intensity of absorption peaks in the range of 0.7 to $1.35 \mathrm{eV}$ exhibited a quadratic dependence on $F_{\text {app }}$ [35]. The experimental data could be well described by a theory introducing electronelectron interactions in the Hamiltonian, thus strengthening the excitonic picture.

Besides the relevance of many-body interactions in SWCNTs, there is growing evidence that the effect of phonons needs also to be considered in the optical absorption process. Inclusion of phonons in the free-electron model leads only to a small distortion in the band structure (figure 8(b)) [38]. In contrast, in the excitonic picture with phonon coupling, a sideband appears apart from the main excitonic absorption peak (figure $8(a)$ ). The existence of exciton-phonon coupling is supported by a recent experiment measuring the photocurrent across an individual s-SWCNT as a function of excitation energy [39]. The resulting photo-current spectrum displayed two peaks at around 1.3 and $1.5 \mathrm{eV}$, as shown in figure 9. Both the resonance and side peak exhibited the same polarization dependence with a maximum when the light polarization was oriented parallel to the tube axis. Comparing the obtained data to theory, the resonance and the side peak were assigned to the excitonic transition corresponding to $E_{22} \mathrm{~s}$ of the investigated s-SWCNT and an exciton state with the simultaneous excitation of a $\mathrm{C}-\mathrm{C}$ bond stretching phonon $(\mathrm{G}$ band), respectively.

Evidence for strong exciton-phonon coupling has furthermore been obtained by low-temperature $(4 \mathrm{~K})$ photoluminescence excitation (PLE) spectroscopy on individual SWCNTs [40]. In addition to purely excitonic transitions associated with $E_{11}^{\mathrm{s}}$, several additional strong bands due to the excitation of one or more phonon modes were observed in this work. Most prominent of these bands was a peak $\approx 0.2 \mathrm{eV}$ higher in energy than the first ground excitonic 
state, which was attributed to the nanotube $G$ band. The intensity of the phonon-assisted modes was of the same order of magnitude as the ground state of the exciton associated with $E_{22}$ s. Furthermore, these modes exhibited significant broadening ( $>35 \mathrm{meV}$ ) indicative of an ultrafast relaxation ( $<10 \mathrm{fs})$, due to the presence of strong exciton-phonon interactions. Similar measurements performed by a different group at room temperature revealed a similar sideband at $\approx 0.2 \mathrm{eV}$ above the first excitonic state, whereas the other phonon modes observed at low temperature were absent [41]. It has been speculated whether the sideband could arise from a higher-lying exciton state without the involvement of phonons [42]. However, time-resolved temperature-dependent PLE spectra obtained from various $(6,4)$ SWCNTs have helped to resolve this issue [43]. The PL decay times of the tubes were found to significantly decrease with temperature, which is in contrast to the existence of purely radiative excitonic decay as proposed by Maultzsch et al [42]. Moreover, the amplitude of the sideband showed a marked decrease at low temperatures. Both observations indicate that the PL is strongly influenced by non-radiative processes. In addition, the PL lifetimes varied from one tube to another for the same chirality $(6,4)$. This finding signifies the presence of different concentrations of localized trap states at tube inhomogeneities, which could also account for the variations in non-radiative exciton decay rates.

\section{Electroluminescence}

The direct band gap of s-SWCNTs coupled with the possibility of both p- and n-type conduction [44, 45] has stimulated the investigation of electrically induced light emission from individual CNTs. Electroluminescence is usually observed at $\mathrm{p}-\mathrm{n}$ junctions, where radiative recombination of electrons and holes takes place within the depletion region. In an individual nanotube, a classical $\mathrm{p}-\mathrm{n}$ junction is difficult to realize since this requires doping of very short segments of nanotubes. However, electrically controllable $\mathrm{p}-\mathrm{n}$ junctions can be created through appropriate control over the drain and gate voltages. Ambipolar s-SWCNT-FETs have been successfully used to this end, as the Schottky barrier width at TiC contacts allows for thermally assisted electron tunnelling through the barriers. In such a device, electrons and holes can be simultaneously injected from the two contacts by choosing a gate voltage that is lower than the drain bias. In such a situation, the gate-induced fields have opposite signs at the source and drain electrodes. The relative numbers of electrons and holes in the channel are determined by their respective injection rates, which can be controlled by the magnitude of the gate voltage. Formation of a $\mathrm{p}-\mathrm{n}$ junction then occurs at the interface between tube regions containing the two types of charge carriers.

Using such an ambipolar s-SWCNT device, electrically induced infrared emission was demonstrated by Misewich et al [46]. The nanotube had a diameter of $\approx 1.4 \mathrm{~nm}$ corresponding to a band gap of $0.75 \mathrm{eV}$. Maximum emission was detected when the gate voltage was exactly half the drain-source bias $\left(V_{\mathrm{ds}}\right)$, confirming that the emission is indeed due to radiative recombination and not due to thermal heating. At $V_{\mathrm{G}}=V_{\mathrm{ds}} / 2$, the numbers of injected holes and electrons are almost equal, since the fields at the source and drain contacts are nearly

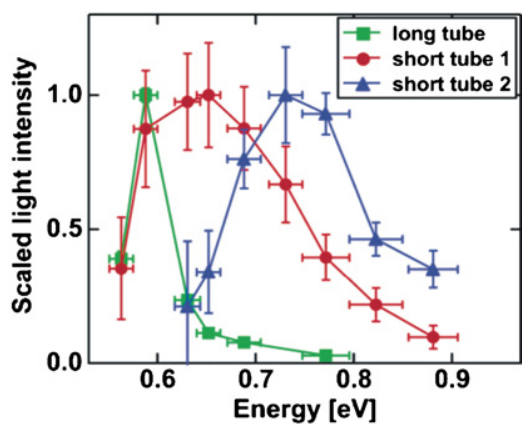

Figure 10. Electroluminescence spectra of three SWCNT-FET devices with varying lengths. Green squares: CVD tube of length $50 \mu \mathrm{m} V_{\mathrm{ds}}=-20 \mathrm{~V}, V_{\mathrm{G}}=-10 \mathrm{~V}, I_{\mathrm{d}}=3.5 \mu \mathrm{A}$; red circles: $500 \mathrm{~nm}$ laser ablation SWCNT, $V_{\mathrm{ds}}=5 \mathrm{~V}, V_{\mathrm{G}}=2.5 \mathrm{~V}, I_{\mathrm{d}}=5 \mu \mathrm{A}$; blue triangles: $500 \mathrm{~nm}$ laser ablation SWCNT, $V_{\mathrm{ds}}=-11 \mathrm{~V}, V_{\mathrm{G}}=$ $-5.5 \mathrm{~V}, I_{\mathrm{d}}=900 \mathrm{nA}$. Reprinted with permission from [47]. (C) 2004 American Chemical Society. The emission was observed by keeping the gate voltage constant and continuously sweeping the drain bias over a range of 0 to $V_{\mathrm{ds}}$ with a time period of 3 to $5 \mathrm{~s}$.

equal but opposite in sign. At lower or higher $V_{\mathrm{G}}$, there is an imbalance in the numbers of carriers resulting in a more unipolar channel and hence a reduced emission intensity. The polarization dependence of the emission was also measured and found to be maximal along the tube axis direction.

Studies on similar ambipolar devices have been utilized to determine the channel length dependence of the spectrum and efficiency of electroluminescence at $V_{\mathrm{G}}=V_{\mathrm{ds}} / 2$ [47]. Long tubes $(\approx 50 \mu \mathrm{m})$ displayed narrow spectral peaks at energies close to the tubes' band gaps, with a spectral width of $\approx 25 \mathrm{meV}$, close to that observed in photoluminescence measurements [48]. Short tubes $(\approx 500 \mathrm{~nm})$, on the other hand, exhibited considerably broader emission peaks as shown in figure 10. Comparing the estimated carrier transit times of $60 \mathrm{ps}$ in long-channel devices to photoluminescence lifetimes which are tens of picoseconds [49], it could be concluded that the electroluminescence in such devices originates from recombination of carriers completely relaxed to the lowest subbands. The larger peak width of $\approx 150 \mathrm{meV}$ for short CNTs (cf figure 10) reflects transit times of around $600 \mathrm{fs}$, which fall within the range of the acoustic phonon scattering times, as has been measured by high-field transport measurements $[50,51]$ and wavelength-dependent pump-probe spectroscopy [49]. Thus the electroluminescence spectra obtained from short-tube devices are characteristic of processes involving hot electrons occurring on the femtosecond time scale. The efficiency of electroluminescence was measured to be around $10^{-6}$ to $10^{-7}$ photons per injected electron-hole pair. This is two to three orders of magnitude smaller than the fluorescence quantum yield measured in photoluminescence experiments $[52,53]$. The low electroluminescence efficiency was attributed to the direct contact of the CNTs with the $\mathrm{SiO}_{2}$ substrate. Nanotubes with larger diameters (smaller band gaps) tend to have lower efficiencies due to non-radiative recombination through multiple phonon emission.

Spatially resolved infrared light emission has been subsequently observed in long-channel ambipolar CNT-FETs [31]. The position of the emissive ambipolar domain was found to be restricted to a small segment of the CNT and could be varied by controlling the gate and drain voltages. A simple 


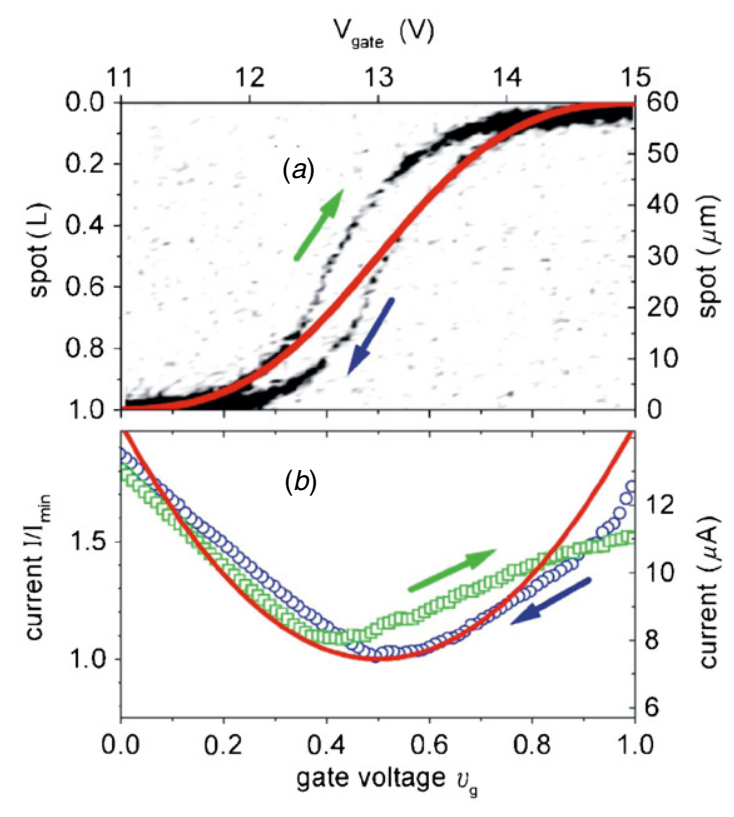

Figure 11. Device characteristics of an electroluminescent s-SWCNT-FET from experiment and theory: $V_{\mathrm{ds}}=15 \mathrm{~V}$.

(a) Position of the light emitting spot as a function of $V_{\mathrm{G}} .0 \mu \mathrm{m}$ corresponds to drain. (b) Gate voltage dependence of the drain current. Top and right scales show absolute units for experimental data; left and bottom scales show scaled units for comparison with theory. In $(a)$ the solid red line is from theory while the grey scale image is from experiment. In $(b)$ circles and squares are data obtained from experiment, while the solid line is from theory. Adapted from [54].

analytical model [54] supported by self-consistent simulations [55] has proven useful to unravel the mechanism underlying the movement of the ambipolar domain. Figure 11(a) reproduces the spatial variation of the IR emission as a function of the gate voltage at a fixed drain bias, from both experiment and theory. Figure 11(b) depicts the corresponding transfer characteristics of the device in the relevant gate voltage range. To understand the movement of the emission spot, one should keep in mind that holes are injected into the CNT channel from the source, while electrons are injected from the drain. At the beginning of the gate voltage sweep (low $V_{\mathrm{G}}$ ), the CNT is in a p-type state with holes as majority carriers. Since there is a very small number of electrons, the $\mathrm{p}-\mathrm{n}$ junction is formed close to the drain. Upon increasing the gate voltage, the emission spot starts shifting away from the drain. In the middle of the gate voltage scan, the carrier densities are balanced at approximately the central region of the tube, from where the emission takes place. Finally, the emission spot is seen on the source side, when the CNT is in an n-type conduction state (at highest $V_{\mathrm{G}}$ ). As apparent from figure 12, the experimentally determined spot position as a function of $V_{\mathrm{G}}$ correlates well with the calculated charge density distribution and the electrostatic potential across the CNT.

In long-channel s-SWCNT devices comprising close-toideal contacts, the drain bias drops completely across the nanotube. This enables the movement of the ambipolar domain between the source and drain by varying $V_{\mathrm{G}}$ between zero and $V_{\mathrm{ds}}$. However, in realistic situations, there is often a sizeable contact resistance at the source and drain. As a consequence,

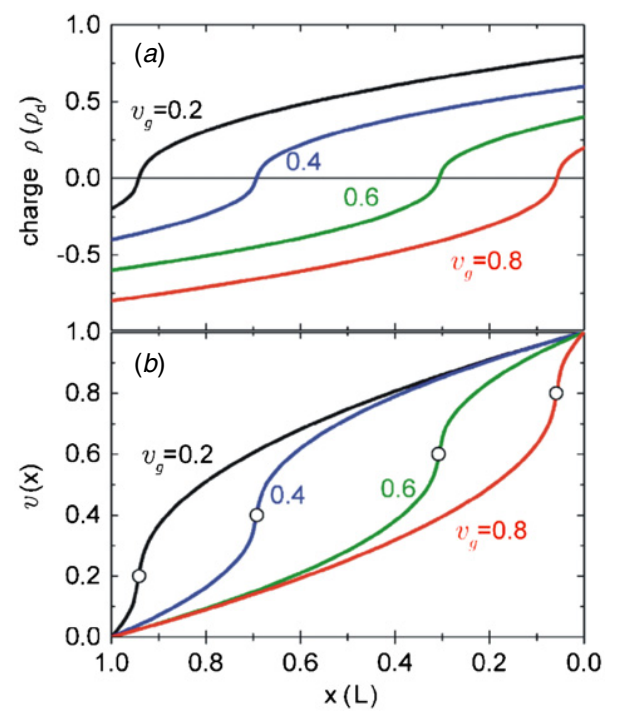

Figure 12. Calculated charge density $(a)$ and electrostatic potential (b) along the nanotube versus normalized channel length (with drain at the right) for fixed $V_{\mathrm{ds}}$ and different values of scaled gate voltage $v_{\mathrm{G}}$. Circles in $(b)$ indicate $v=v_{\mathrm{G}}$ and zero charge density. Adapted from [54].

the gate voltage range over which the emission spot moves is reduced by the total voltage drop at the two contacts. This property opens the possibility of estimating the voltage drop at each of the contacts. A voltage drop of $\approx 5$ to $6 \mathrm{~V}$ has been reported for the sample in figure 11(a), a value consistent with typical threshold voltages observed for CNT Schottky-barrier FETs with a thick gate oxide. In addition to gate-induced movement of the emission spot in the above experiment, additional stationary spots of electroluminescence of varying intensity were observed along the tube. Their origin has been attributed to the creation of minority carriers at defect sites either due to impact ionization or Zener tunnelling.

FET devices composed of a partially suspended CNT channel have led to the discovery of a novel type of electroluminescence caused by unipolar carrier transport [56]. As demonstrated in figure $13(a)$, these devices exhibit IR emission exclusively from the interface between the supported and suspended parts of the CNT, in contrast to fully supported CNTs. A further difference relates to the fact that the emission of the suspended device cannot be controlled by varying the gate voltage. Typical device characteristics are presented in figure 13(b), which reveals that maximum emission occurs under unipolar conditions (at the gate voltage extremities). The drain bias for maximum emission is much lower $(2.5 \mathrm{~V})$, as compared to ambipolar emitters $(\approx 15 \mathrm{~V})$. Added to this, the emission intensity is two to three orders of magnitude larger than that of fully supported samples. Hence, these devices show high electron-to-photon conversion rates close to photoluminescence efficiencies. The following model was proposed to explain the experimental observations: as a consequence of the difference in the dielectric constant between the supported $\left(\mathrm{SiO}_{2}, 200 \mathrm{~nm}\right)$ and suspended (air, $2 \mu \mathrm{m}$ ) parts of the nanotube, the capacitive coupling for the suspended part is reduced. This leads to a strong bending of the CNT bands at the interface where the electrons injected into the conduction band are strongly accelerated. If the accelerated 


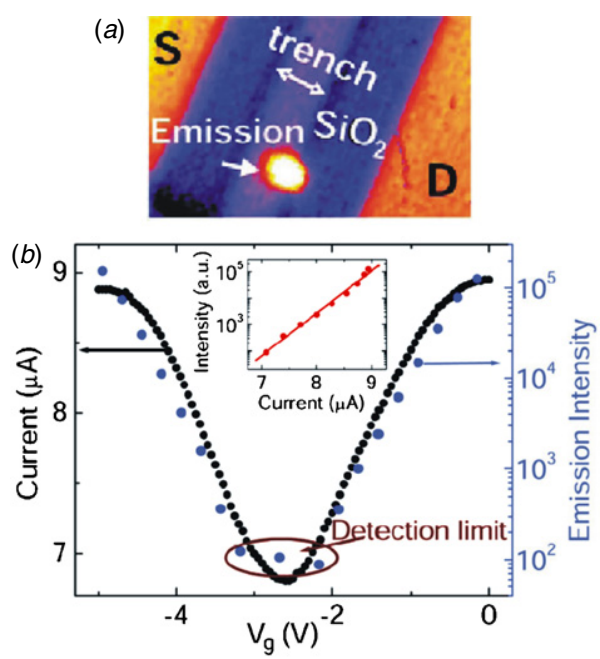

Figure 13. (a) Bright infrared emission seen at the interface between the partially suspended and the supported parts of an individual $\mathrm{CNT}$. The diameter of the CNT is $\approx 2 \mathrm{~nm}$. The total length of the CNT-FET channel is $28 \mu \mathrm{m}$, while the width of the trench is $10 \mu \mathrm{m}$. The oxide thickness is $200 \mathrm{~nm}$ and the trench depth is

$2 \mu \mathrm{m}$. (b) Drain current (blue) and emission intensity (black) from another CNT that is partially suspended as a function of gate voltage at a drain bias of $-5 \mathrm{~V}$. The inset shows that the emission intensity increases exponentially with the drain current. Reprinted with permission from [56]. (C) 2005 AAAS.

electrons reach sufficiently high energies before significant optical phonon scattering sets in, they are able to create an exciton which can subsequently recombine radiatively. For such an impact excitation, the required threshold electric field $\left(E_{\mathrm{th}}\right)$ is theoretically estimated to be around 0.3 to $0.6 \mathrm{MV} \mathrm{cm}^{-1}$ for CNTs. The emission intensity should then be proportional to the impact excitation rate given by $\exp \left(-E_{\mathrm{th}} / E_{\text {int }}\right)$ [57], where $E_{\text {int }}$ is the local field generated at the interface, that is directly proportional to the gate and drain voltages. By fitting the gate-dependent emission curves, $E_{\text {int }}$ was estimated to be 0.01 to $0.2 \mathrm{MV} \mathrm{cm}^{-1}$. The applicability of this model gains support from the observation that the emission intensity increases exponentially with the drain current, as shown in the inset of figure 13(b). By contrast, the emission intensity in fully supported nanotubes is directly proportional to the number of majority or minority carriers.

\section{Photovoltaics}

\subsection{Pristine s-SWCNT devices}

Devices comprising a $\mathrm{p}-\mathrm{n}$ junction commonly exhibit the photovoltaic effect [58]. A p-n junction can be formed in an individual nanotube through electrostatic doping (see previous section), although the operating voltage is quite high. For this purpose, a split-gate architecture is advantageous. In such a configuration, two physically separated gate electrodes allow independent control of the injection rates of charge carriers from the source and the drain. For s-SWCNTs in direct contact with a $\mathrm{Si} / \mathrm{SiO}_{2}$ substrate, complications arise due to trap states at the $\mathrm{SiO}_{2} /$ nanotube interface. However, nanotubes suspended in air enable the fabrication of $\mathrm{p}-\mathrm{n}$ junction diodes

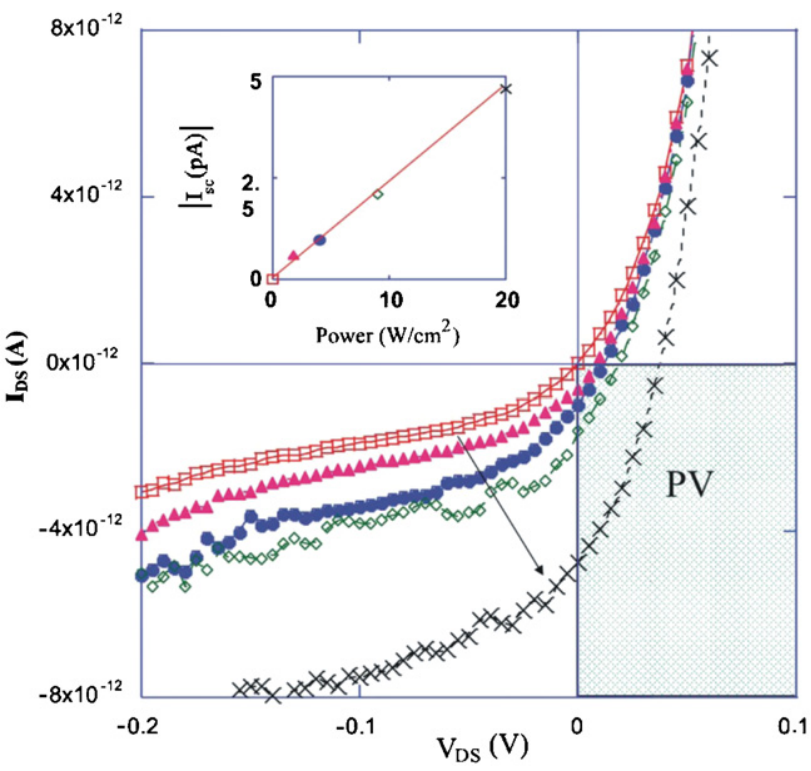

Figure 14. Current-voltage characteristics of an air-suspended split-gated s-SWCNT under illumination from a laser diode at $\lambda_{\text {exc }}=1.5 \mu \mathrm{m}(0.8 \mathrm{eV})$ for various laser intensities. The contacts are asymmetrically gated with $V_{\mathrm{G} 1}=-V_{\mathrm{G} 2}=10 \mathrm{~V}$. The inset shows the dependence of the short-circuit current on the laser intensity. The region of power generation is marked as PV. Adapted from [59].

exhibiting close-to-ideal characteristics [59]. The photocurrent response of such a device illuminated by a laser diode $\left(\lambda_{\text {exc }}=1.5 \mu \mathrm{m}\right)$ at various laser intensities is shown in figure 14. It is evident that with rising laser intensity the short-circuit current increases linearly. Although the estimated power conversion efficiency was only $0.2 \%$, enhanced performance may be achieved in future if smaller tubes with a larger band gap are used.

Photovoltaic devices have also been realized from SWCNT networks implemented into a photo-electrochemical cell [60]. In this study, electron-hole pairs were generated in the SWCNT film through illumination by visible light of $\lambda_{\text {exc }}>$ $400 \mathrm{~nm}$. While the holes were collected by the underlying glass electrode, the electrons were transferred to an acceptor compound contained in the electrolyte solution. The resulting photo-currents were three times higher, when the SWCNTs were deposited on a $\mathrm{SnO}_{2}$-modified glass electrode, due to the improved charge collection efficiency of the high-surfacearea $\mathrm{SnO}_{2}$ layer. The maximum incident photon conversion efficiency (IPCE) at the lowest wavelength was determined to be $0.15 \%$.

\subsection{Nanotube-based hybrid systems}

Apart from the use of pristine SWCNTs for photovoltaic applications, alternative methodologies have been followed aiming at the realization of hybrid systems. One strategy is to combine SWCNTs with polymers into composites, while a second method comprises the chemical functionalization of SWCNTs with photoactive molecules. In photovoltaic devices based upon conducting organic polymers, light harvesting relies upon optical absorption of the $\pi$-conjugated polymer backbones. Dissociation of the generated strongly bound excitons can be accomplished for instance through a 
potential difference across a polymer-metal junction [61]. Alternatively, exciton dissociation can be enhanced by appropriate electron donors or acceptors. The high work function of SWCNTs renders them suitable as p-type dopants for mixing into $\pi$-conjugated polymers. As an additional benefit, the extremely high surface area of the nanotubes ensures a high probability of exciton dissociation. Since the separated carriers need to be transported before they can undergo radiative recombination, it is essential that a sufficiently well-organized percolation network is available, which is best offered by SWCNTs due to their high aspect ratio of $>10^{3}$.

Photovoltaic devices composed of SWCNTs inside a polymer matrix were first demonstrated from a mixed SWCNT/poly(3-octylthiophene) (P3OT) film sandwiched between an indium-tin-oxide (ITO) front contact and aluminium back contact [62]. Device operation involved the dissociation of photo-generated excitons at the interface, followed by efficient transport of holes through the nanotube pathways to the underlying ohmic-contacted Al electrode. Doping the polymer with SWCNTs was found to increase the photo-current by two orders of magnitude and double the open-circuit voltage, as compared to SWCNT-free diodes. Later, alternative SWCNT/polymer photovoltaic devices were realized from poly(2-methoxy-5-( $2^{\prime}$ ethylhexyloxy)-1,4-phenylenevinylene) (MEHPPV) [63] or $\quad \operatorname{poly}\{(m$-phenylene-vinylene $)-c o-[(2,5$-dioctyloxy- $p$ phenylene)vinylene)]\} (PmPV) [64], albeit with lower efficiencies than for the devices incorporating P3OT.

The increasing availability of chemical functionalization schemes for carbon nanotubes [65] has motivated the exploration of the resulting hybrid materials for photovoltaic applications. In such systems, the carbon nanotube acts as an electron acceptor, while the attached organic moieties function as the photo-excitable donor. Both covalent and non-covalent schemes have been pursued to fabricate such donor-acceptor complexes [66]. For SWCNTs with covalently attached pyrene moieties, strong fluorescence quenching without the ability for charge separation has been found [67]. In comparison, SWCNTs covalently modified by ferrocene have been reported to exhibit very slow charge separation dynamics in the order of $4 \mathrm{~ns}$ [68]. Despite the versatility of the covalent approach, the performance of the obtained hybrids seems to be quite poor. The non-covalent approach has been employed to assemble for instance modified pyrene molecules onto the surface of nanotubes by exploiting $\pi-\pi$ interactions. Using a positively charged pyrene derivative in combination with a zinc porphyrin bearing negatively charged side groups, alternate multilayers have been assembled based upon an electrostatic approach [69]. The resulting films, in which the porphyrins act as the primary light absorbers (cf scheme in figure 15), featured fast charge separation coupled with a slow recombination time of around $5 \mu \mathrm{s}$. In this hybrid system, the role of the intermediate pyrene molecules is to decrease the electron-hole recombination probability via separating the donor and acceptor by $\approx 0.6$ nm. The multi-layered architecture reaches monochromatic photon-to-electron conversion efficiencies (IPCE) of up to $\approx 8 \%$, which is about twice the efficiency of single layers of SWCNT/pyrene/porphyrin. It is interesting to note that

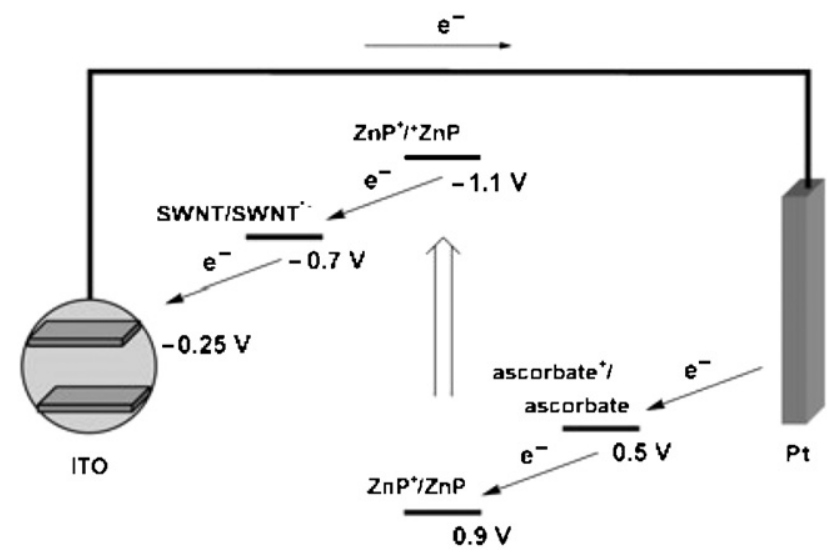

Figure 15. Schematic illustration of photo-current generation in ITO electrodes covered with a single layer of the hybrid material SWCNT/modified pyrene/porphyrin. Adapted from [69].

assemblies incorporating $\mathrm{C}_{60}$ in the place of the SWCNTs exhibited an efficiency of $0.15 \%$, while systems without either $\mathrm{C}_{60}$ or SWCNTs show an IPCE of only $0.08 \%$.

\section{Conclusions and outlook}

SWCNTs have proven promising candidates for a variety of nanoscale optoelectronic devices ranging from photodiodes [59] to electro-optical modulators [70]. However, their applicability in comparison to inorganic nanowires (e.g. $\mathrm{Si}$ [71] or CdS [72]) is still severely limited due to the lack of reliable procedures to selectively produce either metallic or semiconducting tubes. Despite recent progress, the fundamental physics of nanotube interactions with light remains poorly understood. Open questions include the detailed mechanism determining the width of the optical transitions, and the relative contribution of radiative and nonradiative decay channels in photo-excited tubes. Further work is also required towards elucidating the physics of excitons in carbon nanotubes, in particular with regard to their radiative lifetimes [73] and the extent of excitonphonon coupling. During the past 2-3 years, an increasing number of experiments performed at the single-tube-level has contributed to gain a better understanding of these and related issues. For instance, single-nanotube photoluminescence (PL) spectroscopy on a SWCNT-FET has yielded valuable insights into the relation between the recombination lifetime and carrier transit time in nanotubes [74]. Moreover, combined Raman scattering and PL spectroscopy on individual SWCNTs appears well suited to establish a correlation between the emission properties and structural defects in the tubes [75]. Furthermore, Raman studies of electrochemically doped SWCNT films could provide further useful information on the exciton binding energy in SWCNTs [76].

\section{References}

[1] Thostenson E T, Ren Z and Chou T W 2001 Compos. Sci. Technol. 611899

[2] Balasubramanian K and Burghard M 2006 Anal. Bioanal. Chem. 385 online first

[3] Avouris Ph 2004 MRS Bull. 29403 
[4] Cuniberti G, Fagas G and Richter K 2005 Introducing Molecular Electronics (Lecture Notes in Physics vol 680) (Berlin: Springer)

[5] Saito R, Dresselhaus G and Dresselhaus M S 1998 Physical Properties of Carbon Nanotubes (London: Imperial College Press)

[6] Charlier J C and Lambin Ph 1998 Phys. Rev. B 57 R15037

[7] Collins P G, Bradley K, Ishigami M and Zettl A 2000 Science 2871801

[8] Derycke V, Martel R, Appenzeller J and Avouris Ph 2002 Appl. Phys. Lett. 802773

[9] Heinze S, Tersoff J, Martel R, Derycke V, Appenzeller J and Avouris Ph 2002 Phys. Rev. Lett. 89106801

[10] Martel R, Derycke V, Lavoie C, Appenzeller J, Chan K K, Tersoff J and Avouris Ph 2001 Phys. Rev. Lett. 87256805

[11] Chen R J, Franklin N R, Kong J, Cao J, Tombler T W, Zhang Y and Dai H 2001 Appl. Phys. Lett. 792258

[12] Zhao J, Han J and Lu J P 2002 Phys. Rev. B 65193401

[13] Shim M, Back J H, Ozel T and Kwon K W 2005 Phys. Rev. B 71205411

[14] Fujiwara A, Matsuoka Y, Suematsu H, Ogawa N, Miyano K, Kataura H, Maniwa Y, Suzuki S and Achiba Y 2001 Japan. J. Appl. Phys. 40 L1229

[15] Levitsky I A and Euler W B 2003 Appl. Phys. Lett. 831857

[16] Freitag M, Martin Y, Misewich J A, Martel R and Avouris Ph 2003 Nano Lett. 31067

[17] Balasubramanian K, Friedrich M, Fan Y, Wannek U, Mews A, Burghard M and Kern K 2004 Appl. Phys. Lett. 842400

[18] Balasubramanian K, Burghard M, Kern K, Scolari M and Mews A 2005 Nano Lett. 5507

[19] Bube R H 1998 Photovoltaic Materials (London: Imperial College Press)

[20] Bockrath M, Liang W, Bozovic D, Hafner J H, Lieber C M, Tinkham M and Park H 2001 Science 291283

[21] Hunger Th, Lengeler B and Appenzeller J 2004 Phys. Rev. B 69195406

[22] Delaney P, Choi H J, Ihm J, Louie S G and Cohen M L 1998 Nature 391466

[23] Yang C K, Zhao J and Lu J P 2002 Phys. Rev. B 66041403

[24] Kong K, Han S and Ihm J 1999 Phys. Rev. B 606074

[25] Freitag M, Radosavljevic M, Clauss W and Johnson A T 2000 Phys. Rev. B 62 R2307

[26] Rochefort A and Avouris Ph 2002 Nano Lett. 2253

[27] Jiang C, Zhao J, Therese H A, Friedrich M and Mews A 2003 J. Phys. Chem. B 1078742

[28] Tans S J and Dekker C 2000 Nature 404834

[29] Freitag M, Johnson A T, Kalinin S V and Bonnell D A 2002 Phys. Rev. Lett. 89216801

[30] Li Y, Ravaioli U and Rotkin S V 2006 Phys. Rev. B 73035415

[31] Freitag M, Chen J, Tersoff J, Tsang J C, Fu Q, Liu J and Avouris Ph 2004 Phys. Rev. Lett. 93076803

[32] Wang F, Dukovic G, Brus L E and Heinz T F 2005 Science 308838

[33] Zhao H, Mazumdar S, Sheng C X, Tong M and Vardeny Z V 2006 Phys. Rev. B 73075403

[34] Perebeinos V, Tersoff J and Avouris Ph 2004 Phys. Rev. Lett. 92257402

[35] Kennedy J W, Vardeny Z V, Collins S, Baughman R H, Zhao H and Mazumdar S 2005 Preprint cond-mat/0505071

[36] Gadermaier C, Menna E, Meneghetti M, Kennedy J W, Vardeny Z V and Lanzani G 2006 Nano Lett. 6301

[37] Sebastian L and Weiser G 1981 Phys. Rev. Lett. 461156

[38] Perebeinos V, Tersoff J and Avouris Ph 2005 Phys. Rev. Lett. 94027402

[39] Qiu X, Freitag M, Perebeinos V and Avouris Ph 2005 Nano Lett. 5749

[40] Htoon H, O'Connell M J O, Doorn S K and Klimov V I 2005 Phys. Rev. Lett. 94127403

[41] Plentz F, Ribeiro H B, Jorio A, Strano M S and Pimenta M A 2005 Phys. Rev. Lett. 95247401
[42] Maultzsch J, Pomraenke R, Reich S, Chang E, Prezzi D, Ruini A, Molinari E, Strano M S, Thomsen C and Lienau C 2005 Phys. Rev. B 72241402

[43] Hagen A, Steiner M, Raschke M B, Lienau C, Hertel T, Qian H, Meixner A J and Hartschuh A 2005 Phys. Rev. Lett. 95197401

[44] Derycke V, Martel R, Appenzeller J and Avouris Ph 2001 Nano Lett. 1453

[45] Liu X, Lee C, Zhou C and Han J 2001 Appl. Phys. Lett. 793329

[46] Misewich J A, Martel R, Avouris Ph, Tsang J C, Heinze S and Tersoff J 2003 Science $\mathbf{3 0 0} 783$

[47] Freitag M, Perebeinos V, Chen J, Stein A, Tsang J C, Misewich J A, Martel R and Avouris Ph 2004 Nano Lett. 41063

[48] Bachilo S M, Strano M S, Kittrell C, Hauge R H, Smalley R E and Weisman R B 2002 Science 2982361

[49] Ostojic G N, Zaric S, Kono J, Strano M S, Moore V C, Hauge R H and Smalley R E 2004 Phys. Rev. Lett. 92117402

[50] Javey A, Guo J, Paulsson M, Wang Q, Mann D, Lundstrom M and Dai H 2004 Phys. Rev. Lett. 92106804

[51] Park J Y, Rosenblatt S, Yaish Y, Sazonova V, Uestunel H, Braig S, Arias T A, Brouwer P W and McEuen P L 2004 Nano Lett. 4517

[52] Wang F, Dukovic G, Brus L E and Heinz T F 2004 Phys. Rev. Lett. 92177401

[53] Lebedkin S, Hennrich F, Skipa T and Kapper M M 2003 J. Phys. Chem. B 1071949

[54] Tersoff J, Freitag M, Tsang J C and Avouris Ph 2005 Appl. Phys. Lett. 86263108

[55] Guo J and Alam M A 2005 Appl. Phys. Lett. 86023105

[56] Chen J, Perebeinos V, Freitag M, Tsang J, Fu Q, Liu J and Avouris Ph 2005 Science 3101171

[57] Okuto Y and Crowell C R 1972 Phys. Rev. B 63076

[58] Sze S M 1981 Physics of Semiconductor Devices (New York: Wiley)

[59] Lee J U 2005 Appl. Phys. Lett. 87073101

[60] Barazzouk S, Hotchandani S, Vinodgopal K and Kamat P V 2004 J. Phys. Chem. B 10817015

[61] Granstroem M, Petritsch K, Arias A C, Lux A, Andersson M R and Friend R H 1998 Nature 395257

[62] Kymakis E and Amaratunga G A J 2002 Appl. Phys. Lett. 80112

[63] Kazaoui S, Minami N, Nalini B, Kim Y and Hara K 2005 J. Appl. Phys. 98084314

[64] Star A, Lu Y, Bradley K and Gruner G 2004 Nano Lett. 41587

[65] Balasubramanian K and Burghard M 2005 Small 1180

[66] Guldi D M, Rahman G M A, Zerbetto F and Prato M 2005 Acc. Chem. Res. 38871

[67] Georgakilas V, Kordatos K, Prato M, Guldi D M, Holzinger M and Hirsch A $2002 \mathrm{~J}$. Am. Chem. Soc. 124760

[68] Guldi D M, Marcaccio M, Paolucci D, Paolucci F, Tagmatarchis N, Tasis D, Vazquez E and Prato M 2003 Angew. Chem. Int. Edn $\mathbf{4 2} 4206$

[69] Guldi D M, Rahman G M A, Prato M, Jux N, Qin S and Ford W 2005 Angew. Chem. Int. Edn 442015

[70] Wu Z et al 2004 Science 3051273

[71] Ahn Y, Dunning J and Park J 2005 Nano Lett. 51367

[72] Gu Y, Kwak E S, Lensch J L, Allen J E, Odom T W and Lauhon L J 2005 Appl. Phys. Lett. 87043111

[73] Spataru C D, Ismail-Beigi S, Capaz R B and Louie S G 2005 Phys. Rev. Lett. 95247402

[74] Ohno Y, Kishimoto S and Mizutani T 2006 Nanotechnology 17549

[75] Hartschuh A, Qian H, Meixner A J, Anderson N and Novotny L 2005 Nano Lett. 52310

[76] Wang Z, Pedrosa H, Krauss T and Rothberg L 2006 Phys. Rev. Lett. 96047403 